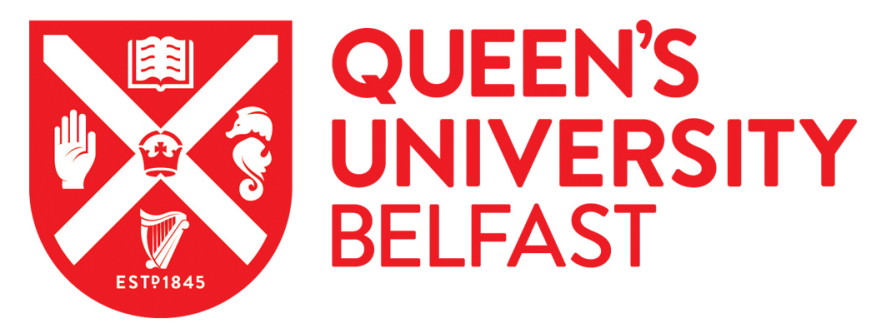

\title{
Investigation Into The Effect of Varying L-leucine Concentration on the product characteristics of Spray-dried Liposome Powders
}

Chen, K-H., Mueannoom, W., Gaisford, S., \& Kett, V. (2012). Investigation Into The Effect of Varying L-leucine Concentration on the product characteristics of Spray-dried Liposome Powders. Journal of Pharmacy and Pharmacology, 64(10), 1412-24. https://doi.org/10.1111/j.2042-7158.2012.01521.x

Published in:

Journal of Pharmacy and Pharmacology

Document Version:

Peer reviewed version

Queen's University Belfast - Research Portal:

Link to publication record in Queen's University Belfast Research Portal

\section{General rights}

Copyright for the publications made accessible via the Queen's University Belfast Research Portal is retained by the author(s) and / or other copyright owners and it is a condition of accessing these publications that users recognise and abide by the legal requirements associated with these rights.

Take down policy

The Research Portal is Queen's institutional repository that provides access to Queen's research output. Every effort has been made to ensure that content in the Research Portal does not infringe any person's rights, or applicable UK laws. If you discover content in the Research Portal that you believe breaches copyright or violates any law, please contact openaccess@qub.ac.uk. 
1 Title: Investigation Into The Effect of Varying L-leucine Concentration on the product

2 characteristics of Spray-dried Liposome Powders

3 K-H Chen ${ }^{\mathrm{a}}$, W. Mueannoom ${ }^{\mathrm{b}}$, S. Gaisford ${ }^{\mathrm{b}}$, V.L. Kett ${ }^{\mathrm{a} *}$

4 a School of Pharmacy, Queen's University of Belfast, Belfast BT9 7BL UK

5 b The School of Pharmacy, University of London, 29/39 Brunswick Square, London WC1N

$6 \quad 1 \mathrm{AX}$

$7 \quad$ *corresponding author email address v.kett@qub.ac.uk

8 Telephone $+44(0) 2890972008$

$9 \quad$ Fax $+44(0) 2890247794$

10

11 
12 Abstract

13 Spray-dried formulations offer an attractive delivery system for administration of drug

14 encapsulated into liposomes to the lung, but can suffer from low encapsulation efficiency

15 and poor aerodynamic properties. In this paper the effect of the concentration of the

16 anti-adherent I-leucine was investigated in tandem with the protectants sucrose and

17 trehalose. Two manufacturing methods were compared in terms of their ability to offer small

18 liposomal size, low polydispersity and high encapsulation of the drug indometacin.

19 Unexpectedly sucrose offered the best protection to the liposomes during the spray drying

20 process, although formulations containing trehalose formed products with the best powder

21 characteristics for pulmonary delivery; high glass transition (Tg) values, fine powder fraction

22 (FPF) and yield. It was also found that L-leucine contributed positively to the characteristics

23 of the powders, but that it should be used with care as above the optimum concentration of

$24 \quad 0.5 \%(\mathrm{w} / \mathrm{w})$ the size and polydispersity index increased significantly for both disaccharide

25 formulations. Relating to the method of manufacture it was found that while both the

26 sucrose and trehalose conferred protection on the liposomes produced using either method

27 the ethanol-based proliposome method offered improved drug incorporation and did not

28 suffer from loss of drug caused by dilution effects.

29 (200 words)

30

31 Keywords. liposomes; spray drying; disaccharide; leucine 


\section{Introduction}

Liposomes are suited to encapsulation of a variety of drugs from small molecular

weight compounds to macromolecules and including both hydrophilic and lipophilic entities.

This is reflected in the range of therapeutics that have been tested in liposomal

formulations including cytotoxic agents [3]; bronchodilators and anti-asthmatics [4; 5];

antibiotics [6] as well as photosensitizing agents [7] and genetic material [8-10]. The use of spray drying to produce stable powder formulations for pulmonary administration is attractive since it offers several advantages over the parenteral route [1]. Aerodynamic diameter is a crucial factor in determining deposition of particles in the different sites of the respiratory tract. Particles in the range 1-6 $\mu \mathrm{m}$ are best suited to deposition in the lower airway following inhalation [2]; those with diameters $>6 \mu \mathrm{m}$ are deposited in the oropharynx, whereas smaller particles $(<1 \mu \mathrm{m})$ are exhaled during normal tidal breathing. In addition, fine particle fraction (FPF, the fraction of powder emitted from the inhaler with a particle size $\leq 5 \mu \mathrm{m})$ is a critical parameter to predict the proportion of the emitted dose that can deliver deeply into the lower respiratory system. The Use of liposomes as carriers offers benefits including protection of drug from enzymatic degradation; prolonging retention time and reducing side effects.

Many methods are available for the manufacture of liposomes, including thin-film

hydration [11], organic solvent injection [12], reverse-phase evaporation [13] and 1 dehydration-rehydration [12; 14]. Both the ethanol injection and proliposome methods of 
52 liposome preparation offer good potential for scale-up and have been used to encapsulate

53 a wide range of substances [15-25] [26-31]. The ethanol injection method involves the

54 rapid injection of a lipid-ethanol solution into an excess of aqueous medium to.

55 spontaneously form large unilamellar vesicles (LUVs) [12]. Advantages of the technique

56 include simplicity and low risk of lipid degradation or oxidation. The ethanol-based

57 proliposome method is based on the preparation of hydrated stacked bilayer sheets in a

58 water-ethanol solution termed proliposomes. Spontaneous formation of liposomal

59 suspensions (multilamellar vesicles, MLVs) is achieved by addition of excess aqueous

60 solution to a lipid mixture [26]. The MLVs produced can be further processed for the

61 preparation of oligolamellar and unilamellar liposomes.

62

63

The effect of disaccharide protectants on the stability of spray dried liposomes has

64 been examined previously [32-34], while amino acids have been shown to play an

65 important role in improving the aerosol behaviour of spray-dried powders by reducing

66 moisture sorption and surface tension of dried particles [37; 38]; they can also protect

67 proteins against thermal stresses and denaturation [39]. Leucine, in particular, tends to

68 improve powder aerosol properties, this anti-adherent effect has been attributed to its

69 hydrophobic character and surfactant-like properties that allow it to migrate rapidly to the

70 surfaces of the particles during drying [40; 41] and it has been shown to interact well with

71 lipid membranes [42]. Two studies have used leucine in combination with sugars at very 
72 high fixed concentrations [43; 44]. To the best of our knowledge, the effect of varying

73 leucine concentration on the spray drying of liposomal systems has not been investigated

74 previously. In this paper we demonstrate the effect of varying concentrations of the

75 anti-adherent L-leucine in combination with optimised concentrations of disaccharides and

76 show for the first time that formulation effects can lead to significant differences in the

77 product characteristics for spray dried liposomes, especially in terms of for sucrose

78 formulations. Furthermore, at higher concentrations leucine increases liposome size.

79 Additionally, we investigated whether switching from the ethanol injection to the

80 proliposome method of liposome preparation affected liposome properties or drug release.

81 Indometacin was chosen as it has been used previously as a model drug in liposomal

82 studies $[45 ; 25]$.

83

84 2. Materials and methods

85

\subsection{Materials}

86 Cholesterol (PhEur grade), sucrose (>99\%), L-Leucine (PhEur grade), $\mathrm{NaCl}(\geq 99 \%)$,

87 Trifluoroacetic acid (99\%), Iron (III) chloride hexahydrate ( $\geq 99 \%)$, Ammonium thiocyanate

88 ( $\geq 99 \%)$, Sephadex G-50 (20-80 $\mu \mathrm{m})$, Indometacin ( $\geq 99 \%)$ phosphotungstic acid hydrate

89 and ethanol ( $\geq 99.5 \%)$ were purchased from Sigma Chemicals. $\alpha, \alpha$-Trehalose Dihydrate

90 (high purity, low endotoxin) was purchased from Ferro Pfanstiehl. Soy phosphatidylcholine

91 (LIPOID S 75, 80\%) and soy phosphatidylcholine (Lipoid S PC, >98\%) were purchased 
92 from Lipoid. Methanol (HPLC grade, >99.8\%) and chloroform (99.2\%) were purchased from

93 VWR. Phosphate buffered saline tablets was purchased from Invitrogen Corporation. All

94 aqueous solutions were prepared with de-ionized water.

95

2.2 Methods

97

\subsubsection{Preparation and drying of liposomal dispersions}

\subsubsection{Preparation of liposomes by ethanol injection}

99

Small unilamellar vesicles (SUV's) were prepared by ethanol injection method followed

by sonication in order to reduce liposomal size. Each batch was prepared on a $10 \mathrm{~g}$ scale.

$0.02 \mathrm{~g}$ Indometacin was dissolved in $0.7 \mathrm{~g}$ ethanol (at $57^{\circ} \mathrm{C}$ ) together with the lipids composed of $1 \mathrm{~g}$ SPC and $0.115 \mathrm{~g}$ cholesterol. Hydration media were prepared by dissolving varying amounts $(2.5,5,7.5,10$ and $15 \% \mathrm{w} / \mathrm{w}$ ) of disaccharides (sucrose or trehalose) in $0.9 \% \mathrm{NaCl}$ solution. Once the optimum concentration of these protectants had been determined, liposomal dispersions were prepared using various concentrations

$(0.25,0.5$ and $1 \% \mathrm{w} / \mathrm{w})$ of L-Leucine added into the hydration medium also containing the optimum concentration of each protectant. The lipid solution was rapidly injected into prepared liposomal suspension was then submitted to a probe sonication process with a sequence of $40 \mathrm{~s}$ of sonication and $20 \mathrm{~s}$ of rest in an ice bath to the desired size. In all 
sonicated liposomes were annealed at $4{ }^{\circ} \mathrm{C}$ overnight before centrifugation $(12,000 \mathrm{rpm}, 30$ $\mathrm{min}$ ) and diluted 4-fold to give a final lipid concentration of $25 \mathrm{mg} / \mathrm{mL}$ of lipid.

\subsubsection{Preparation of liposomes by proliposome method}

$1.5 \mathrm{~g}$ of the appropriate hydration medium was added to a lipid dispersion containing soya lecithin $(750 \mathrm{mg})$, cholesterol $(86.4 \mathrm{mg})$ and indometacin $(30 \mathrm{mg})$ in ethanol $(600 \mathrm{mg})$ at $60{ }^{\circ} \mathrm{C}$. The dispersion was stirred for $10 \mathrm{~min}$ at $60{ }^{\circ} \mathrm{C}$, cooled to room temperature and then converted to a $25 \mathrm{mg} / \mathrm{mL}$ liposome suspension by drop-wise addition of the rest of hydration medium with continuous stirring for $2 \mathrm{hr}$ following sonication and centrifugation.

\subsubsection{Spray drying of liposomes}

Spray drying was performed with a Mini Spray-dryer (Büchi 190). Applied spraying parameters were: inlet temperature $100^{\circ} \mathrm{C}$, outlet temperature $70^{\circ} \mathrm{C}$, air-flow $600-650 \mathrm{NI} / \mathrm{h}$, aspirator setting 20 (100\%), pump setting $2.5-3 \mathrm{ml} / \mathrm{min}$. A 0.5-mm nozzle was used.

\subsubsection{Liposome size analysis}

The average liposome size was determined with a ZetaSizer 3000HS (Malvern Instruments Ltd, Malvern, United Kingdom) at a temperature of $25 \pm 0.1^{\circ} \mathrm{C}$. Samples of the dispersion were diluted with hydration medium and the Z-average vesicle size and polydispersity were determined at $25{ }^{\circ} \mathrm{C}$ by dynamic light scattering. The values of the 
viscosity and refractive index used in the calculation of the liposome size of the light scattering data were modified in terms of protectant concentration. The intensity of the laser light scattered by the samples was detected at an angle of $90^{\circ}$ with a photomultiplier. For each specimen 10 autocorrelation functions were analyzed using a Contin analysis. From this analysis, the z-average diameter $\left(D_{z}\right)$ was obtained, which is an approximation of the diameter of the liposomes. The particle size distribution was characterized using the polydispersity index (PI). The spray-dried liposomes were reconstituted with de-ionized water to attain the original lipid content according to the method of Bligh and Dyer [46] and then performing the Stewart assay [47]. Samples were further diluted with hydration medium for liposome size analysis.

\subsubsection{HPLC analysis of indometacin}

HPLC was carried out using a Waters system (Waters 1525 Binary HPLC Pump, Waters IN-Line Degasser AF, Waters 2487 Dual $\lambda$ Absorbance Detector, Waters 717 plus Autosampler). Luna C-18 column (100A $\mathrm{A}^{0}, 150 \times 4.6 \mathrm{~mm} 5 \mu \mathrm{m}$, Phenomenex) was used. The detector wavelength was set at $260 \mathrm{~nm}$. The mobile phase consisted of a gradient of methanol and $0.1 \% \mathrm{v} / \mathrm{v}$ trifluoroacetic acid. The gradient schedule was: (a) $0-4.5 \mathrm{~min}, 85 \%$ methanol, flow rate of $1 \mathrm{ml} / \mathrm{min}$; (b) 4.5-5.5 $\mathrm{min}, 85 \rightarrow 100 \%$ methanol, flow rate of $1 \mathrm{ml} / \mathrm{min}$; (c) $5.5-7 \mathrm{~min}, 100 \%$ methanol, flow rate of $1.2 \mathrm{ml} / \mathrm{min}$; (d) $7-9 \mathrm{~min}, 100 \%$ methanol, flow rate of $1.5 \mathrm{ml} / \mathrm{min}$; (e) $9-15 \mathrm{~min}, 100 \%$ methanol, flow rate of $1.5 \mathrm{ml} / \mathrm{min}$; (f) $15-16 \mathrm{~min}$, 
$152100 \%$ methanol, flow rate of $1 \mathrm{ml} / \mathrm{min}(\mathrm{g}) 16-17 \mathrm{~min}, 100 \rightarrow 85 \%$ methanol, flow rate of 1.0 $153 \mathrm{ml} / \mathrm{min}$; (h) $17-23 \mathrm{~min}, 85 \%$ methanol, flow rate of $1.0 \mathrm{ml} / \mathrm{min}$. In order to determine 154 encapsulation efficiency (EE \%) $50 \mu \mathrm{L}$ of separate liposome solutions and reconstituted 155 dispersions were applied to a Sephadex G-50 column and eluted with hydration medium. 156 The fractions were diluted with methanol and the concentrations of indometacin were 157 determined by HPLC. Loading efficiency was determined by dividing the encapsulated drug 158 content $(\mu \mathrm{g})$ by the lipid content $(\mathrm{mg})$. The encapsulation efficiency (EE $(\%))$ of indometacin 159 was determined from the ratio of encapsulated to total drug concentration.

\subsubsection{Water content and thermal analysis of the powders.}

Thermogravimetric studies were carried out to measure the water content of the spray-dried liposomes using a TGA (Q500, TA instruments). Samples were heated from 20 and heat capacity). The product was sealed into a hermetic aluminium pan and after reported as mean values $(n=4-6)$ with standard deviation. 
173 morphology was observed using a scanning electron microscope (JEOL 6500F field

174 emission scanning electron microscope).

\subsubsection{Powder particle size analysis}

The volume mean diameter of spray-dried liposomes was measured by Laser Light

Diffraction Analyzer (HELOS/BR, Sympatec, Clausthal-Zellerfeld, Germany).

Approximately $5 \mathrm{mg}$ of powder were suspended in chloroform in a $50 \mathrm{ml}$ glass cuvette and

stirred with a magnetic bar at $1000 \mathrm{rpm}$. A short period of sonication (60 s) at a power of

60W (CUVETTE, Sympatec; 8.5 mm diameter ultrasound tip) was applied before sizing [48;

49]. A R4 lens was used allowing measurements in the range of $0.45-875 \mu \mathrm{m}$.

\subsection{7 in vitro release of indometacin from liposomes}

Free drug was removed from the reconstituted liposome suspensions by use of

7.4 PBS release medium in a shaking incubator $\left(37^{\circ} \pm 0.5 \circ \mathrm{C}, 60 \mathrm{rpm}, 25 \mathrm{~mm}\right.$ throw;

drug release determined using the HPLC method. The release volume was kept constant

throughout.

\subsubsection{Aerodynamic study}

A twin-stage liquid impinger was used to determine the emitted dose and fine particle 
192 fraction (FPF) of the spray-dried powders. The dried liposome powders were filled into

193 number 3 gelatin capsules. A dry powder inhaler (Cyclohaler, Teva, UK) was attached to 194 the mouthpiece of emitted dose apparatus. Ten capsules were pierced and the dried 195 liposome powders were emptied at $60 \mathrm{~L} / \mathrm{min}$ for 10 seconds. The powders deposited at 196 each stage were washed out and recovered. The powders deposited in the inhaler and 197 capsules were also collected. After being diluted with methanol to a suitable concentration, 198 each indometacin solution was assayed by HPLC. The FPF values were defined as the 199 powder mass recovered at stage 2. The results are expressed as the percentage of drug 200 dose emitted to the capsule content (loaded dose).

201 2.2.9 Statistical analysis:

202 Kruskal-Wallis analysis with Dunn's all pairwise multiple comparisons or Mann-Whitney $203 U$ test were used to calculate the $p$ values using SigmaPlot 8 software. Differences were 204 deemed significant if $p$ values were $<0.05$

205

206 3. Results and discussion

3.1Initial Determination of Optimum Disaccharide and L-leucine concentrations

\subsubsection{Effect of disaccharide concentration on liposomal size and PI}


212 Initially the optimum concentration of either sucrose or trehalose as protectants during the

213 spray drying process was determined.

$214 \quad<$ Figure 1>

As shown in Figure 1a, the addition of $2.5 \%(\mathrm{w} / \mathrm{w})$ sucrose to the hydration medium did not prevent the aggregation and fusion of reconstituted liposomes after spray drying, but

217 increasing the concentration to $5 \%(\mathrm{w} / \mathrm{w})$ inhibited liposomal size increase prior to spray

218 drying and after reconstitution more effectively. A further increase to $7.5 \%(\mathrm{w} / \mathrm{w})$ showed no

219 significant change in the PI values. 10\% (w/w) Sucrose was more protective still; this was

220 the only formulation containing disaccharide alone that showed no statistical difference in

221 liposomal size after drying. A further increase in concentration to $15 \%(w / w)$ resulted in an

222 increase in liposome size. This effect can also be seen by comparing the $S_{\mathrm{F}} / S_{\mathrm{I}}$ ratios,

223 where is $S_{F}$ is the final liposomal size after rehydration and $S_{\text {I }}$ is the initial liposomal size

224 [50]. Previous research into freeze-drying of liposomes demonstrated that solute

225 incorporated by liposome in the presence of lyoprotectant in a mass ratio of sugar: lipid of

$2262: 1$ could be effectively retained after freeze-drying [51], in the $5 \%(\mathrm{w} / \mathrm{w})$ sucrose

227 dispersions the mass ratio of sucrose to lipid is close to this ratio. A similar effect was

228 observed when trehalose was used as the protectant (Figure 1b). However, the protective

229 effect of trehalose was not so good; the addition of $15 \%(w / w)$ trehalose dihydrate to the 230 hydration medium did not prevent the liposome size increasing after reconstitution $(p<$ $2310.05)$ 
234 The recovery rate of the spray-dried powders significantly improved when increasing the

235 concentration of protectants from $2.5 \%(\mathrm{w} / \mathrm{w})$ to $5 \%(\mathrm{w} / \mathrm{w})$ for both sugars (Figure 2), 236 although further addition of either disaccharide showed little additional effect. Further, the 237 spray-dried liposomes with trehalose had a much higher recovery rate than those with 238 sucrose, which is ascribed to its higher $\operatorname{Tg}\left(101^{\circ} \mathrm{C}\right.$ compared with $64^{\circ} \mathrm{C}$ for sucrose [36]. 239 The sticky point (Ts) of an amorphous powder is generally considered to lie $10-20{ }^{\circ} \mathrm{C}$ above $240 \mathrm{Tg}$; if the outlet temperature of the dryer surpasses Ts then particle cohesion increases 241 sharply and there may also be increased adhesion to the dryer walls [35]. Because the 242 powder temperature would have been greater than the Tg for the sucrose formulation, the 243 particles would have been more prone to adherence to the walls of the spray-dryer. 244 Increasing the concentration of either protectant reduced the water content of the 245 spray-dried powders (Figure 2). Since Tg data are inversely related to water content this 246 would be expected. Based on the results in 3.1.1 and 3.1.2 the formulations containing $10 \%$ 247 sucrose and $15 \%$ trehalose were selected for further optimisation by addition of the 248 anti-adherent L-leucine.

$249<$ Figure 2>

$250<$ Figure 3> 


\subsubsection{Effect of L-leucine concentration on liposomal size}

Figure $3 a$ indicates that the inclusion of L-Leucine at a concentration of $0.5 \%(w / w)$ was the most effective in preventing size changes for both the $10 \%(\mathrm{w} / \mathrm{w})$ sucrose and the $15 \%(w / w)$ trehalose formulations, with no statistical difference seen in the sizes prior to spray drying compared with the reconstituted dispersions. Increasing the concentration to $1 \%(w / w)$ L-Leucine had the effect of markedly increasing the liposomal size in the reconstituted dispersions. This might be caused by partitioning of the hydrophobic amino acid into the lipid membrane during drying, causing vesicle fusion. While this phenomenon has not been investigated for spray dried liposomes it has been observed previously during freezing and freeze-drying of liposomes; Anchordoguy et al [52] found that the amino acids with hydrocarbon side chains increased membrane damage during freeze/thaw trials. Popova et al [53] observed that the amphiphilic aromatic amino acids tryptophan and phenylalanine induced solute leakage and membrane fusion during freezing studies of liposomes, while Mohammed [54] et al demonstrated that basic, polar amino acids stabilized liposomes during lyophilisation but that at higher concentrations these amino acids promoted vesicle fusion.

$$
<\text { Figure 4> }
$$

\subsubsection{Effect of L-leucine concentration on recovery rate and water content}

Addition of $0.5 \%(\mathrm{w} / \mathrm{w})$ L-Leucine markedly improved the recovery rate of the $10 \%(\mathrm{w} / \mathrm{w})$ sucrose formulations (Figure 4) but had little effect on the liposomes formulated with 15\% 
$272(w / w)$ trehalose, which exhibited good yields already. L-Leucine did not have a significant

273 effect on the water contents of the powders (Figure 4) but a marked improvement in the

274 appearance of the powders was apparent to the naked eye. Formulations without L-leucine

275 were clumped into aggregates in the $\mathrm{mm}$ size range while increasing L-leucine reduced the

276 size. This effect was especially apparent for the sucrose formulations.

277 Taking all data from 3.1 and 3.2 into account the two formulations selected for further

278 testing were those containing $0.5 \%(w / w)$ leucine and either $10 \%(w / w)$ sucrose or $15 \%$ $279(w / w)$ trehalose.

\subsection{Effect of Liposome manufacturing method on liposomal size and drug content}

Once the optimal levels of disaccharide and anti-adherent had been determined, the size was clearly larger for the formulations prepared by the proliposome method. It has been suggested that the proliposome-prepared liposomes produce multilamellar vesicles

[31], while those prepared by the ethanol-injection method are primarily unilamellar, which are more amenable to size reduction under the same sonication conditions. Based on encapsulation efficiency and loading efficiency, the two methods have the same capability to incorporate indometacin. However, lipid loss during the alcohol injection method could not be avoided owing to this process involving injection of lipid solution into hydration 
292 liposome size reduction using a high-pressure homogenizer, whereby the liposomal size 293 and range decreased with increasing concentration of ethanol [55].

294 Statistically significant changes were seen between freshly prepared and reconstituted 295 liposomes prepared by the ethanol injection in terms of PI value, encapsulated content and 296 loading efficiency. The small increases in encapsulated content and loading efficiency were 297 attributed to unentrapped drug being reincorporated into the liposomes during 298 reconstitution, as has previously been demonstrated for liposomes prepared using the DRV 299 (dried-rehydrated vesicle) method [56]. Even though the formulation was optimised using 300 the ethanol injection method, excellent encapsulation efficiency in liposomes prepared by 301 the proliposome method was obtained. Dispersions produced using the proliposome 302 method showed small statistically significant reductions in PI values for both formulations, 303 while for the trehalose formulation there was also a small statistically significant reduction in 304 liposomal size after drying. However, the liposomal indometacin content and its loading 305 efficiency did not significantly change during spray drying. In short, while sucrose enabled a 306 better loading efficiency when comparing samples produced by the ethanol injection 307 method; the combination of either $10 \%(w / w)$ sucrose and $0.5 \%(w / w)$ leucine or $15 \%(w / w)$ 308 trehalose and $0.5 \%(\mathrm{w} / \mathrm{w})$ leucine in the formulations could effectively protect liposomes 309 prepared by either method against spray drying stress. In terms of drug loading and 310 efficiency liposomes prepared by the proliposome method incorporated drug more 311 effectively than those produced using the ethanol-injection method (Table 1). In addition, a 
312 dilution effect was observed when comparing loading efficiency of liposomal stock solutions

313 and the final dispersions prepared by the ethanol-injection method, which was attributed to

314 encapsulated drug leaking out of the liposome stock solutions upon dilution as observed by.

315 Foldvari et al [57]. TEM images (data not shown) indicate that the vesicular structure of 316 liposome in the presence of the optimised disaccharide and anti-adherent formulations 317 could be preserved very well through spray drying .

$319<$ Figure 5>

\subsection{Imaging, size analysis and aerodynamic properties of the spray-dried powders}

SEM analysis showed that particles of the optimally formulated sucrose powder

wrinkled (Figure 5d, e and f), which potentially prevents particles from adhering tightly to each other, thus preventing aggregation and lowering the energy required to disperse them [58]. This may explain why the sucrose formulation appeared to contain more aggregates. Furthermore, the fine particle fraction was higher for the trehalose/L-leucine formulation

327 than for that containing sucrose/L-Leucine (Figure 6).

$328<$ Figure 6>

329 Bosquillon et al [59] had reported that the type of sugar incorporated did not affect 330 morphology of the spray-dried powders. The wrinkled surface perhaps can be attributed to 331 the inclusion of additives that alter the surface tension that controls droplet shape during 
drying as Adler et al [60] demonstrated that surfactant could change the interface viscosity

333 and that consequently, the dried particle morphology also changed. By increasing the ratio

334 of surfactant to other additives, more spherical, smooth particles were obtained. It is

335 proposed that L-leucine at a concentration of $0.5 \%(\mathrm{w} / \mathrm{w})$ functions as a surfactant. Hence,

336 it is suggested that the ratio of $0.5 \%(\mathrm{w} / \mathrm{w})$ leucine to $15 \%(\mathrm{w} / \mathrm{w})$ trehalose is below the

337 critical point of powder morphology conversion between roughness and smoothness and

338 so it tends to exhibit a wrinkled surface. The formulation containing $10 \%(\mathrm{w} / \mathrm{w})$ sucrose

339 and $0.5 \%(\mathrm{w} / \mathrm{w})$ exhibited a smoother morphology. To investigate whether this was a

340 concentration effect rather than being specific for trehalose, spray dried liposomes were

341 prepared with a sucrose concentration of $15 \%(w / w)$, but these still had smooth surfaces

342 (Figure $5 c$ and $d$ ), indicating that the wrinkling is not caused by surface tension effects

343 alone. The SEM images also show that the diameters of all the spray dried powders were

344 less than $10 \mu \mathrm{m}$ (Figure 5). Accurate size distribution data are given in Table 2, which show

345 that all formulations the volume mean diameters were measured to be $3 \sim 4 \mu \mathrm{m}$ with the

346 exception of the formulation containing $10 \%(\mathrm{w} / \mathrm{w})$ sucrose and $0.5 \%(\mathrm{w} / \mathrm{w})$ L-leucine,

347 prepared by ethanol injection, which gave a larger diameter of $5.40 \mu \mathrm{m}$. This size increase

348 cannot be attributed to hygroscopicity of the sucrose formulations [34] as the water content

349 values for all formulations were similar. Although the sucrose formulations exhibited lower

$350 \mathrm{Tg}$ values than the trehalose formulations, as might be expected, there was no significant

351 difference between those obtained for the liposomes prepared by the ethanol injection 
352 rather than the proliposome method. The emitted dose and fine particle fraction of 353 spray-dried liposome formulated with trehalose and L-leucine were higher than those 354 formulated with 10\% (w/w) sucrose and 0.5\% (w/w) L-leucine. (Figure 6). Therefore, spray 355 dried liposomes with 15\% (w/w) trehalose and $0.5 \%(\mathrm{w} / \mathrm{w})$ leucine exhibited better aerosol 356 powder performance than those in the presence of $10 \%(\mathrm{w} / \mathrm{w})$ sucrose and $0.5 \%(\mathrm{w} / \mathrm{w})$ 357 leucine in terms of emitted dose, aerodynamic diameter and fine particle fraction.

$358<$ Figure $7>$

$359 \quad 3.5$ Release of indometacin from reconstituted liposomes

360 Release data for Indometacin from reconstituted liposomes for both optimised 361 formulations using both methods of manufacture are shown in Figure 7. Diffusion of free 362 indometacin through the dialysis membrane was measured as a control. The release profile 363 of free drug shows over $80 \%$ diffusion in $4 \mathrm{hr}$. Over the same interval, the reconstituted 364 liposomes prepared by the ethanol-injection method released $15.1 \%$ and $16.9 \%$ drug 365 (optimised formulations containing sucrose \& trehalose respectively) and released $\sim 45 \%$ of 366 the encapsulated drug over $24 \mathrm{hr}$. Those prepared by the proliposome method in the 367 presence of disaccharide (sucrose or trehalose) and leucine exhibited drug release of $36821.8 \%$ and $24.3 \%$ in $4 \mathrm{hr}$ and in total released $54.5 \%$ and $61.5 \%$ over 24 hours respectively

369 (Figure 7). These differences are not significant suggesting that the mode of release is the 370 same for all formulations. The mechanism responsible for the release of drug from the 371 liposomes may be due to diffusion phenomena, degradation effects, or a combination of 
372 both processes. To examine the drug release kinetics and mechanism, the release data

373 were fitted to models representing zero-order, first-order, and Higuchi's square root of time

374 and the Korsmeyer-Peppas models (Table 3). All systems showed best correlation with the

375 Higuchi model and anomalous (non-Fickian) diffusion $(n>0.5)$. It is reasonable to 376 propose that this is because the indometacin is located within the phospholipid membrane 377 of the liposomes and must diffuse through in order to be released.

4. Conclusions

The inclusion of either $10 \%(\mathrm{w} / \mathrm{w})$ sucrose or $15 \%(\mathrm{w} / \mathrm{w})$ trehalose dihydrate and $0.5 \%$

$381(\mathrm{w} / \mathrm{w})$ L-leucine protected liposomes prepared by ethanol injection or proliposome method against spray drying stress in terms of size change, polydispersity index, encapsulated drug content and loading efficiency of the reconstituted liposomes. Formulation design was of more importance than the method of liposome manufacture. The method for preparing the liposomes had no effect on the stability or encapsulation efficiency of spray-dried liposomes with optimal protectant and anti-adherent. as an additive for spray dried liposomes in combination with disaccharide. While there was a clear advantage in using this material at an optimum level; at higher concentrations it caused an increase in liposomal size upon rehydration of the spray dried powders that might be attributed to it partitioning into the lipid membrane during drying, causing vesicle 
392 fusion. This is an area for future research.

393

394 
References

[1] Cryan SA. Carrier-based strategies for targeting protein and peptide drugs to the lungs. Aaps Journal 2005; 1: E20-E41.

[2] Zeng XM et al. The controlled delivery of drugs to the lung. Int J Pharm 1995; 2: 149-164.

[3] Mccullough HN, Juliano RL. Organ-selective action of an anti-tumor drug pharmacologic studies of liposome-encapsulated beta-cytosine arabinoside administered via the respiratory system of the rat. J Natl Cancer Inst 1979; 3: 727-731.

[4] Mccalden Ta AR, And Mihalko Pj. Bronchodilator efficacy of liposome formulations of metaproterenol sulfate in the anesthetized guinea pig. Journal of Liposome Research 1989; 2: 211-222.

[5] Taylor KMG et al. The influence of liposomal encapsulation on sodium cromoglycate pharmacokinetics in man. Pharm Res 1989; 7: 633-636.

[6] Wong JP et al. Liposome delivery of ciprofloxacin against intracellular francisella tularensis infection. J Control Release 2003; 3: 265-273.

[7] Ferro S et al. Efficient photoinactivation of methicillin-resistant staphylococcus aureus by a novel porphyrin incorporated into a poly-cationic liposome. Int J Biochem Cell Biol 2007; 5: 1026-1034.

[8] Guo WJ et al. Characterization of a novel diolein-based Ipdii vector for gene delivery. $J$ Control Release 2002; 1 : 121-132.

[9] Igarashi S et al. Biosurfactant mel-a enhances cellular association and gene transfection by cationic liposome. J Control Release 2006; 3: 362-368. 
418 [10] Maitani $Y$ et al. Cationic liposome (dc-chol/dope=1 : 2) and a modified ethanol injection 419 method to prepare liposomes, increased gene expression. Int J Pharm 2007; 1-2: 33-39.

420 [11] Bangham AD, Standish, M.M., Watkins, J.C., . Diffusion of univalent ions across the 421 lamellae of swollen phospholipids J Mol Biol 1965: 238-252.

422 [12] Batzri S, Korn ED. Single bilayer liposomes prepared without sonication. Biochim 423 Biophys Acta 1973; 4: 1015-1019.

424 [13] Szoka F, Papahadjopoulos D. Procedure for preparation of liposomes with large 425 internal aqueous space and high capture by reverse-phase evaporation. Proc Natl Acad Sci 426 U S A 1978; 9: 4194-4198.

427 [14] Kirby CJ, Gregoriadis G. Preparation of liposomes containing factor viii for oral 428 treatment of haemophilia. J Microencapsul 1984; 1: 33-45.

429 [15] Naeff R. Feasibility of topical liposome drugs produced on an industrial scale. 430 Advanced Drug Delivery Reviews 1996; 3: 343-347.

431 [16] Both DM et al. Liposome-encapsulated ursolic acid increases ceramides and collagen 432 in human skin cells. Arch Dermatol Res 2002; 11: 569-575.

433 [17] Wagner A et al. Liposomes produced in a pilot scale: Production, purification and 434 efficiency aspects. Eur J Pharm Biopharm 2002; 2: 213-219.

435 [18] Elzainy AaW et al. Hydroxyzine from topical phospholipid liposomal formulations:

436 Evaluation of peripheral antihistaminic activity and systemic absorption in a rabbit model. $437 \quad$ AAPS pharmSci 2003; 4: E28.

438 [19] Stano P et al. Novel camptothecin analogue (gimatecan)-containing liposomes 439 prepared by the ethanol injection method. Journal of Liposome Research 2004; 1-2: 
441 [20] Justo OR, Moraes AM. Kanamycin incorporation in lipid vesicles prepared by ethanol 442 injection designed for tuberculosis treatment. J Pharm Pharmacol 2005; 1: 23-30.

443 [21] Ugwu S et al. Preparation, characterization, and stability of liposome-based 444 formulations of mitoxantrone. Drug Dev Ind Pharm 2005; 2: 223-229.

445 [22] Zhong HJ et al. Multivesicular liposome formulation for the sustained delivery of 446 breviscapine. Int J Pharm 2005; 1-2: 15-24.

447 [23] Padamwar MN, Pokharkar VB. Development of vitamin loaded topical liposomal 448 formulation using factorial design approach: Drug deposition and stability. Int J Pharm 2006; 449 1-2: 37-44.

450 [24] Wang Z et al. Development and pharmacokinetics of nimodipine-loaded liposomes. $J$ 451 Pharm Pharmacol 2006; 9: 1289-1294.

452 [25] Jaafar-Maalej C et al. Ethanol injection method for hydrophilic and lipophilic 453 drug-loaded liposome preparation. Journal of Liposome Research 2010; 3: 228-243.

454 [26] Perrett S et al. A simple method for the preparation of liposomes for pharmaceutical 455 applications - characterization of the liposomes. J Pharm Pharmacol 1991; 3: 154-161.

456 [27] Turanek $\mathrm{J}$ et al. Linkup of a fast protein liquid chromatography system with a stirred 457 thermostated cell for sterile preparation of liposomes by the proliposome-liposome method: 458 Application to encapsulation of antibiotics, synthetic peptide immunomodulators, and a 459 photosensitizer. Anal Biochem 1997; 2: 131-139.

460 [28] Pavelic Z et al. Liposomes containing drugs for treatment of vaginal infections. Eur J $461 \quad$ Pharm Sci 1999; 4: 345-351. 
462 [29] Rengel RG et al. High efficiency entrapment of superoxide dismutase into

463 mucoadhesive chitosan-coated liposomes. Eur J Pharm Sci 2002; 5: 441-448.

464 [30] Ishikawa $\mathrm{H}$ et al. Liposomal microcapsulation of enzymes by proliposome method with 465 chitosan-coating. Journal of the Faculty of Agriculture Kyushu University 2005; 1: 141-149.

466 [31] Elhissi AMA et al. Formulations generated from ethanol-based proliposomes for 467 delivery via medical nebulizers. J Pharm Pharmacol 2006; 7: 887-894.

468 [32] Hauser H, Strauss G. Stabilization of small unilamellar phospholipid-vesicles during 469 spray-drying. Biochim Biophys Acta 1987; 2: 331-334.

470 [33] Goldbach P et al. Spray-drying of liposomes for a pulmonary administration .1.

471 Chemical-stability of phospholipids. Drug Dev Ind Pharm 1993; 19: 2611-2622.

472 [34] Lo YL et al. Liposomes and disaccharides as carriers in spray-dried powder

473 formulations of superoxide dismutase. J Control Release 2004; 2-3: 259-272.

474 [35] Maury M et al. Effects of process variables on the powder yield of spray-dried trehalose 475 on a laboratory spray-dryer. Eur J Pharm Biopharm 2005; 3: 565-573.

476 [36] Hatley RH. Glass fragility and the stability of pharmaceutical preparations--excipient 477 selection. Pharm Dev Technol 1997; 3: 257-64.

478 [37] Seville PC et al. Amino acid-modified spray-dried powders with enhanced 479 aerosolisation properties for pulmonary drug delivery. Powder Technology 2007; 1: 40-50.

480 [38] Lechuga-Ballesteros D et al. Trileucine improves aerosol performance and stability of 481 spray-dried powders for inhalation. J Pharm Sci 2008; 1: 287-302.

[39] Arakawa T et al. Biotechnology applications of amino acids in protein purification and 483 formulations. Amino Acids 2007; 4: 587-605. 
484 [40] Lucas $\mathrm{P}$ et al. Enhancement of small particle size dry powder aerosol formulations 485 using an ultra low density additive. Pharm Res 1999; 10: 1643-1647.

486 [41] Chew NYK et al. Effect of amino acids on the dispersion of disodium cromoglycate 487 powders. J Pharm Sci 2005; 10: 2289-2300.

488 [42] Oradd G et al. Effects of peptide hydrophobicity on its incorporation in phospholipid 489 membranes - an nmr and ellipsometry study. Biochimica Et Biophysica $490 \quad$ Acta-Biomembranes 2011; 1: 244-252.

491 [43] Chougule $\mathrm{M}$ et al. Nano-liposomal dry powder inhaler of tacrolimus: Preparation, 492 characterization, and pulmonary pharmacokinetics. International Journal of Nanomedicine $493 \quad 2007 ; 4: 675-688$.

494 [44] Chougule $\mathrm{M}$ et al. Development of spray dried liposomal dry powder inhaler of dapsone. 495 Aaps Pharmscitech 2008; 1: 47-53.

496 [45] Puglia C et al. Evaluation of in-vivo topical anti-inflammatory activity of indometacin 497 from liposomal vesicles. J Pharm Pharmacol 2004; 10: 1225-1232.

498 [46] Bligh EG, Dyer W. J. . A rapid method of total lipid extraction and purification. Can J $499 \quad$ Biochem Phys 1959; 8: 911-917.

500 [47] Stewart JCM. Colorimetric determination of phospholipids with ammonium 501 ferrothiocyanate. Anal Biochem 1980; 1: 10-14.

502 [48] Dalpiaz A et al. Developement and characterization of biodegradable nanospheres as 503 delivery systems of anti-ischemic adenosine derivatives. Biomaterials 2005; 11: 1299-1306.

504 [49] Minne A et al. Optimization of the aerosolization properties of an inhalation dry powder 505 based on selection of excipients. Eur J Pharm Biopharm 2008; 3: 839-844. 
506 [50] Abdelwahed W et al. Investigation of nanocapsules stabilization by amorphous

507 excipients during freeze-drying and storage. Eur J Pharm Biopharm 2006; 2: 87-94.

508 [51] Crowe JH, Crowe LM. Factors affecting the stability of dry liposomes. Biochim Biophys 509 Acta 1988; 2: 327-334.

510 [52] Anchordoguy T et al. Mechanisms of interaction of amino-acids with

511 phospholipid-bilayers during freezing. Biochim Biophys Acta 1988; 2: 299-306.

512 [53] Popova AV et al. Differential destabilization of membranes by tryptophan and

513 phenylalanine during freezing: The roles of lipid composition and membrane fusion.

514 Biochimica Et Biophysica Acta-Biomembranes 2002; 1: 109-118.

515 [54] Mohammed AR et al. Amino acids as cryoprotectants for liposomal delivery systems. 516 Eur J Pharm Sci 2007; 5: 406-413.

517 [55] Rodriguez RB, Xamani MS. Liposomes prepared by high-pressure homogenizers. $518 \quad$ Liposomes, Pt A 2003: 28-46.

519 [56] Zadi B, Gregoriadis G. A novel method for high-yield entrapment of solutes into small 520 liposomes. Journal of Liposome Research 2000; 1: 73-80.

521 [57] Foldvari M et al. Topical liposomal local-anesthetics - design, optimization and 522 evaluation of formulations. Drug Dev Ind Pharm 1993; 19: 2499-2517.

523 [58] You Y et al. Physical characteristics and aerosolization performance of insulin dry 524 powders for inhalation prepared by a spray drying method. J Pharm Pharmacol 2007; 7: $525927-934$.

526 [59] Bosquillon $\mathrm{C}$ et al. Influence of formulation excipients and physical characteristics of 527 inhalation dry powders on their aerosolization performance. J Control Release 2001; 3: 
528 329-339.

529 [60] Adler M et al. Surface composition of spray-dried particles of bovine serum

530 albumin/trehalose/surfactant. Pharm Res 2000; 7: 863-870.

531

532

533 
Table 1. The characterization of liposomes prepared by ethanol injection and by proliposome. The hydration buffers were in either a combination of $10 \%(\mathrm{w} / \mathrm{w})$ sucrose and $0.5 \%(\mathrm{w} / \mathrm{w})$ L-Leucine or $15 \%(\mathrm{w} / \mathrm{w})$ trehalose dihydrate and $0.5 \%(\mathrm{w} / \mathrm{w})$ L-Leucine. ${ }^{*}$ denotes $\mathrm{P}<0.05$ (Mann-Whitney $\mathrm{U}$ test) in comparison with the corresponding samples prior to spray-drying. $+\mathrm{P}<0.05$ (Mann-Whitney $U$ test) compared to formulations with the inclusion of trehalose/L-Leucine. Each value represents the mean \pm SD (three different batches) and $n$ denotes replicate measurements of each batch.

\begin{tabular}{|c|c|c|c|c|}
\hline \multirow[t]{2}{*}{ Preparative methods } & \multicolumn{2}{|c|}{ Ethanol injection } & \multicolumn{2}{|c|}{ Proliposome } \\
\hline & $\begin{array}{c}\text { Before } \\
\text { spray-drying }\end{array}$ & $\begin{array}{c}\text { After } \\
\text { reconstitution }\end{array}$ & $\begin{array}{c}\text { Before } \\
\text { spray-drying }\end{array}$ & $\begin{array}{c}\text { After } \\
\text { reconstitution }\end{array}$ \\
\hline \multicolumn{5}{|c|}{ Liposome with $10 \%(w / w)$ sucrose and $0.5 \%(w / w)$ L-Leucine } \\
\hline $\begin{array}{l}\text { Liposome size }(\mathrm{nm}) \& \\
\text { (PI) }\end{array}$ & $\begin{array}{l}107.4 \pm 13.8 \\
(0.20 \pm 0.02)\end{array}$ & $\begin{array}{l}115.1 \pm 11.5^{*} \\
\left(0.25 \pm 0.04^{*}\right)\end{array}$ & $\begin{array}{c}137.9 \pm 4.9 \\
(0.48 \pm 0.02)\end{array}$ & $\begin{array}{c}134.0 \pm 8.2 \\
\left(0.39 \pm 0.03^{*}\right)\end{array}$ \\
\hline Total drug content $(\mu \mathrm{g} / \mathrm{mL})$ & $407.5 \pm 7.9$ & $384.8 \pm 7.3^{*}$ & $846.4 \pm 23.5$ & $797.2 \pm 13.1^{*}$ \\
\hline $\begin{array}{l}\text { Encapsulation } \\
\text { efficiency }(\%)\end{array}$ & $33.3 \pm 6.1$ & $53.2 \pm 15.1^{*}$ & $45.4 \pm 1.0$ & $45.9 \pm 2.3$ \\
\hline $\begin{array}{l}\text { Encapsulated drug content } \\
\qquad(\mu \mathrm{g} / \mathrm{mL})\end{array}$ & $135.6 \pm 24.9$ & $204.8 \pm 58.1^{*}$ & $397.6 \pm 8.3$ & $366.3 \pm 23.7$ \\
\hline Lipid content $(\mathrm{mg} / \mathrm{mL})$ & $23.57 \pm 0.37$ & $21.60 \pm 1.17^{*}$ & $26.75 \pm 0.47$ & $25.87 \pm 0.26$ \\
\hline $\begin{array}{l}\text { Loading efficiency } \\
\text { ( } \mu \text { g drug/ mg lipid) }\end{array}$ & $5.7 \pm 1.0$ & $9.5 \pm 2.9 *^{+}$ & $14.4 \pm 0.6$ & $14.3 \pm 1.8$ \\
\hline
\end{tabular}

Liposome with $15 \%(w / w)$ trehalose dihydrate and $0.5 \%(w / w)$ L-Leucine

$\begin{array}{ccccc}\text { Liposome size }(\mathrm{nm}) \& & 130.7 \pm 2.7 & 132.7 \pm 4.2 & 138.5 \pm 4.8 & 127.3 \pm 3.6^{*} \\ & (0.31 \pm 0.03) & \left(0.36 \pm 0.02^{*}\right) & (0.52 \pm 0.04) & \left(0.40 \pm 0.03^{*}\right)\end{array}$

(PI) $\begin{array}{llll}(0.31 \pm 0.03) & (0.36 \pm 0.02 *) & (0.52 \pm 0.04) & (0.40 \pm 0.03 *)\end{array}$

\begin{tabular}{|c|c|c|c|c|}
\hline Total drug content $(\mu \mathrm{g} / \mathrm{mL})$ & $442.1 \pm 7.4$ & $428.2 \pm 10.9 *$ & $899.9 \pm 8.1$ & $868.6 \pm 16.7^{*}$ \\
\hline Encapsulation efficiency (\%) & $37.4 \pm 6.4$ & $42.5 \pm 3.8$ & $49.7 \pm 7.1$ & $52.0 \pm 7.0$ \\
\hline $\begin{array}{l}\text { Encapsulated drug content } \\
\qquad(\mu \mathrm{g} / \mathrm{mL})\end{array}$ & $165.3 \pm 27.4$ & $181.8 \pm 16.5$ & $447.5 \pm 67.7$ & $449.1 \pm 66.0$ \\
\hline Lipid content (mg/mL) & $26.21 \pm 0.70$ & $25.51 \pm 1.80$ & $29.60 \pm 0.10$ & $27.85 \pm 0.39$ \\
\hline $\begin{array}{l}\text { Loading efficiency } \\
\text { ( } \mu \text { g drug/ mg lipid) }\end{array}$ & $6.3 \pm 0.9$ & $7.2 \pm 0.70$ & $15.1 \pm 2.2$ & $15.7 \pm 2.0$ \\
\hline
\end{tabular}

